\title{
TROMBOSIS DEL SENO LONGITUDINAL EN EL LACTANTE
}

\author{
Drs. WERNER BUSTAMANTE, LUIS MORENO y GUSTAVO MIRANDA \\ Cátedra de Pediatria del Prof, A. Ariztía. Hospital "Luis Calvo Mackenna".
}

\section{Introducción}

Una de las afecciones de difícil diagnóstico en el lactante y de pronóstico sombrío, es sin duda alguna, la trombosis del seno venoso longitudinal. Su semejanza can diferentes afecciones cerebrales y la gran variabilidad sintomática que ella presenta es motivo para que muchas veces pase desapercibida por el médico, siendo por lo tanto un hallazgo de autopsia.

Su-frecuencia es difícil de precisar debido a que algunos casos pueden presentarse sin dar gran sintomatologia. Holt " al referirse a las trombosis venosas cerebrales, menciona que en Baltimore la han encontrado entre el $1 \%$ y $2 \%$ de las autopsias. Según Hempelmann el seno longitudinal sería uno de los que con menos frecuencia se compromete.

Si recordamos la circulación venosa cerebral, veremos que los principales vasos se encuentran situados en el espesor de la duramadre y se les denomina según su ubicación en:

a) Sero Longitudinal Superior o Sagital, que se encuentra en el borde convexo de la hoz del cerebro y se extiende desde la región frontal hasta la protuberancia occipital interna (prensa de Herófilo), donde desemboca en el seno lateral.

b) Seno Longitudinal Inferior, ocupa el borde cóncayo de la hoz del cerebro en su mitad posterior y desemboca en la extremidad anterior del seno recto.

c) Seno Recto, abicado en la base de la hoz del cerebro.

d) Seno Lateral o Sigmoídeo, que se extiende desde la protuberancia occipital interna (prensa de Herófilo) hasta la base de la porción petrosa del temporal.

e) Seno Cavernoso, situado a cada lado de la silla turea.

Existen numerosos senos más que no entraremos a detallar (s. petroso sup. e inf.; s. occipital, etc.).

Según Ford ${ }^{i}$ la ligadura de un seno venoso o una trombosis de ella pueden no dar grandes síntomas. Esto se explica por la rica red anastomótica que presentan los vasos venosos cerebrales. Cuando la ligadura o trombosis compromete también la desembocadura de algunos vasos colaterales, entonces la circulación de retorno se compromete produciendo edema, congestión y hasta hemorragia, lo cual puede traducirse en aumento de la presión intracraneal y presencia de sangre en el L.C.R.

En general, se describen dos formas clínicas: el tıpo Primario o Marasmático y el tipo Séptico o Secundario. En la prácríca es posible observar, como dice Aschaif :2, que los llamados trombos marasmáticos no son de origen único, y lo mismo que todos los demas trombos, contienen germenes con mucho más frecuencla de lo que antes se suponia.

En el tipo Primario o Marasmático pueae aparentemente no existir una inreccion, motivo por el cual se le denomina también no séptico. Hay tres factores de importancia que favorecen la instalación ae esta clase de trombos:

1. El daño endotelial venoso producido por las toxinas bacterianas a otros venenos.

2. Los cambios que se producen en la sangre y que favorecen la coagulación, como ser: aumento de la viscosidad; hemoconcentración, etc.

3. El retardo de la circulación sanguínea debido a trastornos cardiacos, en especial lo abservado en las cardiopatías congénitas, insuficiencia cardíaca, etc.

Todos estos factores son posibles de encontrar en numerosos cuadros clínicos del lactante, especialmente en las afecciones nutritivas y diarreicas graves, con deshidratación; enfermedades crónicas caquectizantes; policitemias; anemias, etc.

El tipo Séptico o Secundario, es mucho más frecuente que el anterior. Se produce por extensión directa de la infección circunvecina siendo el oído medio, mastoides, huesos craneales, etc., los sitios de donde puede originarse la infección de partida. También se mencionan las enfermedades infecciosas, cuadros septicémicos generales, procesos supurativos, como tambien las punciones o inyecciones en los senos ${ }^{4}$. En casos de procesos sépticos distantes, la trombosis puede afectar cualquier seno cerebral. 
TABM. X.

SINTOMATOLOGIA DE LA TROMBOSIS DEL SENO LONGITUDINAL (Segan Ford)

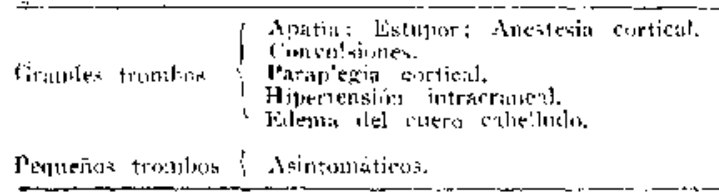

En el lactante es más frecuente observar el tipo primario o marasmático. En general su sintomatología depende del sitio y extensión del proceso. Los trombos de pequeña magnitud pueden no dar sintomatología; en caso contrario y siempre que se comprometa las vías anastomóticas, el cuadro puede iniciarse en forma brusca o lenta, indicando el compromiso neurológico con pérdida de la conciencia. convulsiones, signos de hipertensión craneana, vómitos, cefalea, delirio, ete. A veces se observa edema e ingurgitación venosa del cuero cabelludo.

Christophe ${ }^{7}$ señala que en presencia de crisis frecuentes $y$ subintrantes de epilepsia bruscamente aparecidas en enfermos con antecedentes de infección facial, amigdaljana o mastoidea, hay que pensar en la tromboflebitis cerebral. Michell: menciona la trombosis del seno longitudinal como una de las causantes de la bemiplejia infantil aguda, cuyo compromiso cortical haría por intermedio de las venas cerebrales superficial media. En resumen, podemos decir que el cuadro clinico nada tiene de especifico, existe una variabilidad sintomatica que traduce la localización de la lesión, de acuerdo con los vasos y tejidos comprometidos.

En cuanto a los exámenes de laboratorios, el estudio del líquido eéfalo-raquídeo es el que puede servir de cierta ayuda.
Se menciona que la presión está aumentada, signo al cual algunos autores" le dan importancia. El aspecto es con frecuencia hemorrágico o xantocrómico, con glóbulos rojos en el sedimento. Las proteínas pueden estar atumentadas. Ebbs ${ }^{11}$ considera de interés el aumento que experimentan los cloruros.

El diagnóstico de la trombosis del seno longitudinal en el lacatnte es difícil por las razones ya expuestas. Sin embargo, dobemos pensar en ellas cuando se presentan signos de compromiso neurológico, con hipertensión intracraneana, convulsiones, etc., y con alteraciones del L. C.R., niños con trastornos nutritivos crónicos, deshidratados, sepsis, enfermedades caquécticas o infecto-contagiosas, etc. En su diagnóstico diferencial no debemos ol. vidar la hemorragia cerebral, como también las encefalitis, meningitis y abscesos cerebrales.

Igual que su sintomatología, la evolución de esta afección es muy irregular, pero en general de corta duración. Su pronóstico depende de la magnitud y extensión de la zona trombosada.

En su tratamiento están indicados los antibióticos, cuando se sospeche un probable origen infeccioso. Algunos autores usan la heparina. Cuando la trombosis ya se ha producido, el uso de antocoagulante solo sirve para evitar un mayor progreso del daño. En este sentido la heparina no solo estimula la disolución del coágulo, sino que serviría también para la rápida fusión del edema. El uso del Dicumarol en las trombosis craneanas entraña el peligro de hemorragia. Según Nelson " estos medicamentos son de dudoso resultado. Van Cled " relata un caso de trombosis del seno longitudinal que cede al tratamiento con heparina. El diagnóstico fué

İAIsT.A X: 2.

TROMEOSIS DEL SENO LONGITUDINAL EN FL LACTANTE RESUMEN DE 6 OBSERYACIONES

\begin{tabular}{|c|c|c|c|c|c|c|}
\hline Observación & 1 & 2 & 3 & -4 & 5 & 6 \\
\hline Edad & 2 menes & 5 nieses & 24 nuese: & & 22 110 & 19 thas \\
\hline Sexo & 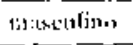 & mase:tius, & mas:ulưv" & 19 meies & ntasculine & trtesculinut \\
\hline Epoca del añol & Invierno & Otinite. & J'rimavera & trumertiflu & Verano & primaver.l \\
\hline $\begin{array}{l}\text { Diagnóstico } \\
\text { al ingre9o }\end{array}$ & $\begin{array}{l}\text { Jjstinfia } \\
\text { bisj:epsia }\end{array}$ & $\begin{array}{l}\text { bistruiti: } \\
\text { thisjepsia }\end{array}$ & $\begin{array}{l}\text { Dixtrotia } \\
\text { lis. netidn. }\end{array}$ & 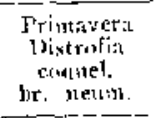 & $\begin{array}{c}\text { Ibistrnii: } \\
\text { Tlre, mi'iar } \\
\text { :nenjngitis } \\
\text { Tbe. }\end{array}$ & $\begin{array}{l}\text { Toxisodis } \\
\text { pre. חentill. } \\
\text { sejoxis }\end{array}$ \\
\hline $\begin{array}{l}\text { Tiempo de } \\
\text { hospitalización }\end{array}$ & 2 dias & I lin & i lists & 7. clias & $2 \mathbf{t}$ dias & 1 ' \\
\hline
\end{tabular}


TABLA No 3 .

SINTOMATOLOGIA OBSERVADA EN LOS 6 CASOS DE TROMBOSIS DEL SENO LONGITUDINAL EN EL LACTANTE

Sirtcmatología:

\begin{tabular}{|c|c|c|c|c|c|c|}
\hline Obaervacion & 1 & 2 & 3 & 4 & 5 & 6 \\
\hline Contienzo & Brufco & Brusco & Lento & Brusce & Lento & Lento \\
\hline Fietrie & + & - & + & + & + & $T$ \\
\hline rérdida del conocimiento & + & + & - & + & + & + \\
\hline Cunvulaiones & + & + & - & + & + & 一 \\
\hline Hipertonia & + & + & $\because$ & + & + & + \\
\hline Nistagirulus & - & - & $\leftarrow$ & + & $\dashv-$ & - \\
\hline Reflejos & + & - & - & + & + & + \\
\hline Rigiclez nutca & $+\div+$ & Nocmal & Normal & $?$ & $?$ & $?$ \\
\hline Font. ant. & Tensid & Sotmal & Normal & $\cdots$ & - & $?$ \\
\hline Ingurgitacioin vetonsa c. c. & - & -- & - & - & $\because$ & - \\
\hline
\end{tabular}

confirmado meses más tarde por la autopsia, donde se encontró el trombo permeabilizado.

La oportunidad de haber reunido seis casos fatales de trombosis del seno longitudinal en el lactante, de los cuales hemos atendido a tres de ellos y conocido la evolución de tres más, nos ha permitido hacer una revisión acerca de este tema. En la Iiteratura pediátrica nacional no hemos encontrado ninguna publicación relacionada con este tipo de trastorno y en la literatura extranjera no son muy abundantes las referencias que hemos podido tener a nuestro alcance.

Nuestra revisión abarca los últimos 10 años $\mathrm{y}$ un total de 2,000 autopsias realizadas por el Servicio de Anatomía Patológica del Hospital de Niños Luis Calvo Mackenna, donde hemos podido reunir los seis casos que motivan el presente trabajo. Su frecuencia sería airededor del $0.3 \%$. Es muy posible, como lo mencionan algunos autores ${ }^{1.0}$, que algunos trombos no alcancen a producir síntomas y que después se hagan permeables, sin alcanzar a la mesa de autopsia.

\section{MATERIAL DE ESTUDTO}

En obsequio a la brevedad hemos consignado en tablas las principales características de nuestras observaciones. Estudiando en conjunto las seis observaciones, hemos podido comprobar la predilección de este trastorno por el lactante distrófi$\mathrm{co}$, ya que todos ellos eran menores de dos años $\mathrm{y}$ con deficiente estado nutritivo.
En cuanto al sexo, encontramos cinco varones y una sola del sexo femenino. La frecuencia estacional correspondió a un caso en Otoño, tres en Primavera, uno en Verano y otro en Invierno.

Los diagnóstico de ingreso y que motivaron la hospitalización de nuestros enfermitos fueron en especial las afecciones del aparato respiratorio y digestivo, anotándose: Dispepsia y Neumonía; Bronconeumonía coqueluchosa; Dispepsia; Bronconeumonia; Tbc. Miliar y Meningitis; Toxicosis y Bronconeumonia. La pasibilidad diagnóstica de una trombosis venosa cerebral, se planteó clínicamente frente a la evolución del caso $\mathrm{N}$ ? 1 y la de una hemorragia cerebral en la observación $N^{9} \mathbf{b}$.

En cuanto a la sintomatologia observa$\mathrm{da}$, vemos que esta corresponde a la de una complicación meningo-encefálica. En tres casos el comienzo fué brusco y en otros tres se presentó en forma solapada, lenta. Uno de nuestros pacientes, el caso Ne 3, no presentó síntomas para sospechar siquiera en este tipo de complicaciones. Lo que observamos con más frecuencia fué la pérdida del conocimiento, fiebre alta, rigidez de la nuca, e hipertonía, síntomas que se presentaron en cinco casos. Convulsiones tuvieron cuatro enfermitos y de variada intensidad. Solo en un caso pudimos comprobar la ingurgitación venosa del cuero cabelludo. En general el diagnóstico de trombosis venosa es difícil y más aún el tratar de precisar la localización en el seno longitudinal.

Una vez establecida la complicación cerebral, pudimos ver que su evolución 


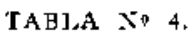

EARACTERISTICAS DEL L.C.R, EN 6 CASOS DE TROMBOSIS DEL SENO LONGITUDINAL EN EL LACTANTE

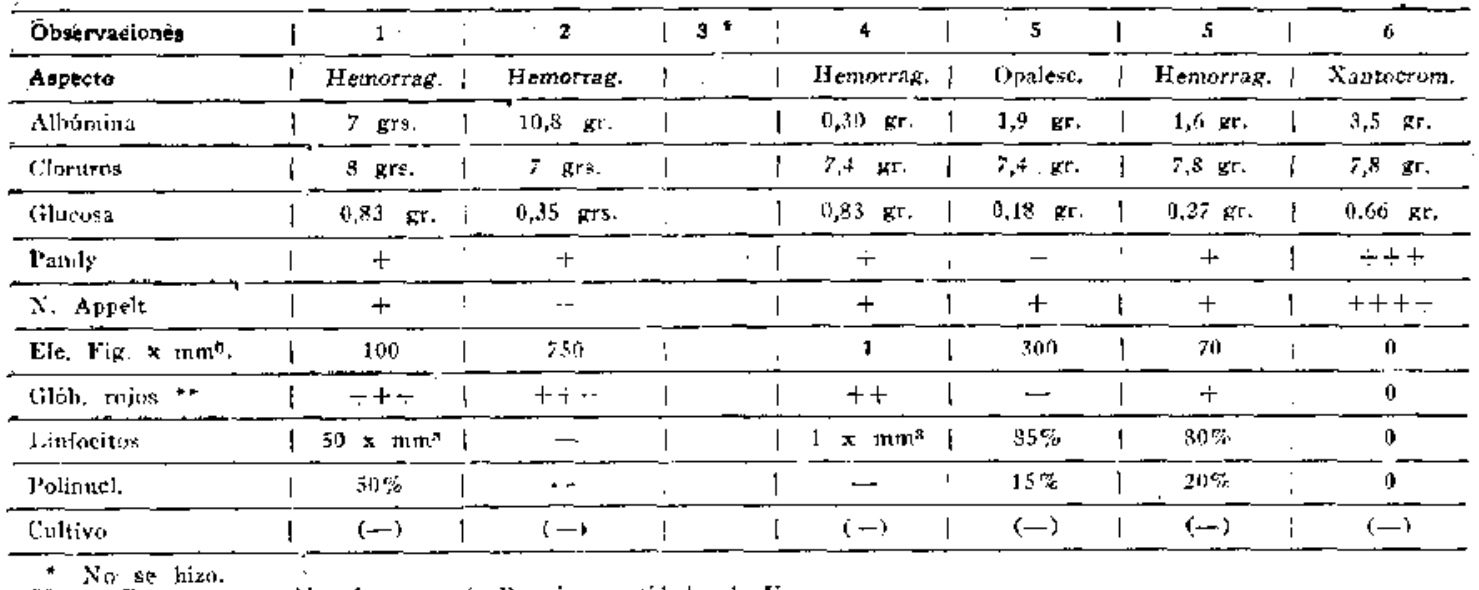

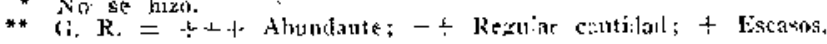

fué siempre de intensa gravedad y fatal. El tiempo de hospitalización varió entre 24 horas y 21 días. Tres de ellos sólo alcanzaron a permanecer $\mathbf{4 8}$ horas en el servicio. Dos estuvieron entre seis a siete días hospitalizados y un caso de Meningitis Tbe estuvo 21 días.

Es interesante señalar las características del L.C.R. E1 aspecto sanguinolento con glóbulos rojos al examen microscópico lo encontramos en cuatro casos. La presion del L.C.R. estuvo ligeramente aumentada en tres casos y normal en dos. Debemos mencionar que no usamos el aparato de Claude para medir la presión y sólo nos guiamos por la salida del líquicio. La albúmína se registró alta en casi todos los casos, con valores que fluctuaron entre 0,30 grs. y 10,8 grs. Los cloruros en general estuvieron dentro de las cifras normales, lo que está en desacuerdo con lo descrito por Ebbs ${ }^{11}$. En cuanto a la glucosa, ésta se presentó alta en tres casos y baja en dos. Las reacciones de Pandy y Monne Appelt fueron siempre positivas. Hubo aumento de los leucocitos en tres observaciones, siendo normal en dos. Los cultivos del L.C.R. fueron todos negativos.

De acuerdo con lo encontrado en nuestros exámenes, estimamos que la presencia de un L.C.R. hemorrágico, con albúminas altas, cloruros normales, reacciones de Pandy y Nonne Appelt positivas, pueden ser de alguna ayuda para orientar al diagnóstico clínico, sin pretender de ningún modo considerarlo como característico de la trombosis venosa cerebral ni de su localización en el seno longitudinal.
En cuanto al estudio anátomo-patológico, pudimos observar, en general, que el compromiso de los vasos venosos de la duramadre fué de carácter intenso. Solamente en tres casos esta lesión estuvo de preferencia localizada en el seno longitudinal; en los restantes el trastorno se extendía y comprometía además otros senos como el lateral, recto y pequeños vasos venosos que desembocan en el seno longitudinal.

En cinco casos encontramos junto a las trombosis venosas una hemorragia cerebral de variada intensidad y localizada especialmente en el sitio más afectado por la trombosis. Esta hemorragia no la encontramos en la observación $\mathrm{N}^{\circ} \mathbf{5}$, donde solo

TABLA N 5 .

PRINCIPALES HALLAZGOS ANATOMOPATOLOGI. COS OBSERVADOS FN 6 CASOS DE TROMBOSIS DEL SENO LONGITUDINAL

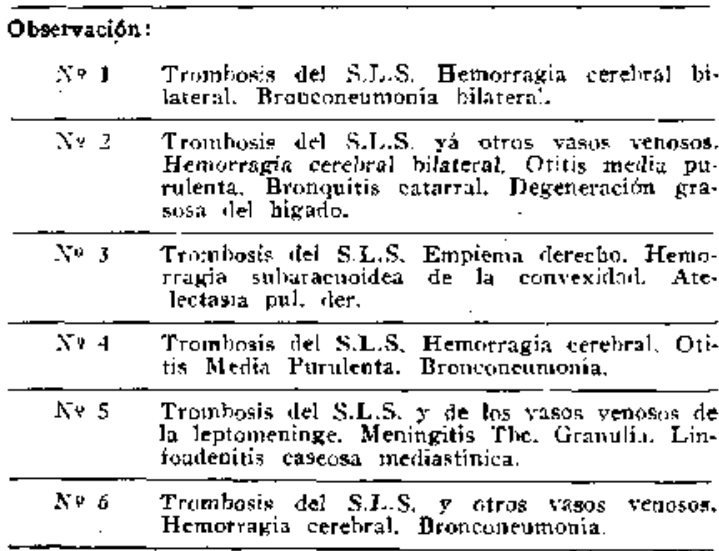


apreciamos la trombosis del seno longitudinal $\mathrm{y}$ una meningitis tuberculosa.

Otros hallazgos anátomopatológicos fueron las bronconeumonías, que se encontraron en tres casos; otitis media purulenta, en dos casos; un empiema y una meningitis tuberculosa.

En general, el estudio histológico de la trombosis localizada en el seno longitudinal revela microscópicamente una masa trombótica que obstruye el lumen vascular.

Esta masa presenta una zona de fijación que toma contacto íntimo con la pared vascular, dando lugar a una verdadera soldadura entre trombo y pared comprometiendo el endotelio que, por lo tanto, queda incluído en la sinfisis.

La masa trombótica misma está constituída por una rica y delicada red de fibrina; cuyas mallas se hallan repletas por acúmulos de hematíes $\mathrm{y}$ polinucleares dispuestos en forma irregular.

\section{COMENTARIO}

De acuerdo con los hallazgos anátomopatológicos, estimamos que nuestros casos corresponden al tipo de trombosis secundarias, ya que hemos encontrado diversos focos sépticos. No obstante, nos ha llamado la atención que de un total de 2.000 autopsias revisadas esta lesión haya correspondido a lactantes distróficos. Por lo que sin duda alguna el factor desnutrición debe desempeñar un papel de importancia en su desencadenamiento, como ya lo mencionamos al hablar de las trombosis de tipo marasmático. Por otra parte, estamos de acuerdo con Hempelman ${ }^{3}$ al estimar la rareza con que se presenta el compromiso del seno longitudinal.

Sabemos que la trombosis venosa puede afectar a cualquier seno cerebral y hay algunos de ellos, como el cavernoso y lateral, que poseen una rica sintomatología clínica que permite sospechar su lesión. En cuanto a la trombosis del seno longitudinal que presentaron nuestros enfermitos, no nos fué posible obtener una sintomatología clínica más especifica para orientarnos a dicho diagnóstico, hecho que explicamos en parte por el hallazgo de otros trastornos anátomopatológicos, como ser la hemorragia cerebral. Es muy posible que en las trombosis producidas por aquellas afecciones de carácter agudo exista una mayor facilidad para las hemorra- gias, siendo esta tendencia mucho menor en los procesos crónicos de tipo meningeo, tal como lo observamos al tratar el caso No 5.

Es interesante señalar la evolución seguida por el caso $\mathrm{N}^{\circ} 3$ que en ningún momento dió una sintomatología para sospechar en un compromiso meningoencefálico, lo que confirmaría el hecho relatado por los textos en el sentido de que a veces la trombosis del seno longitudinal puede ser una sorpresa de autopsia.

\section{RESUMEN}

Se dan a conocer seis observaciones de trombosis venosa con compromiso del seno longitudinal en lactantes distróficos. El cuadro clínico demuestra la dificultad diagnóstica con que tropieza el médico, la que muchas veces pasa desapercibida, siendo por lo tanto un hallazgo de autopsia. Entre los sintomas más llamativos figura la pérdida del conocimiento, fiebre alta, hipertonía generalizada, convulsiones etc. El L.C.R. con frecuencia presenta aspecto hemorrágico, albúmina alta, cloruros normales, Pandy y Nonne Appelt positivo, sin ser característico de esta afección.

En cuanto al estudio anátomopatológico, se comprobó un intenso compromiso de la red venosa cerebral, acompañada en cinco casos de hemorragia cerebral de variada magnitud, lo que en parte enmascara también el diagnóstico.

\section{SUMMARY}

The report of six observations of venous thrombosis involving the longitudinal sinus in distrophic infants is given. Clinical diagnosis is rather difficult and can be unsuspected during life.

In five cases the sinus thrombosis was secondary to respiratory, otic or intestinal infections, and in one case to tuberculous meningitis.

Among the most striking symptoms there was loss of conciousness, high temperature, generalized convulsions, etc. Spinal fluid often presents an hemorrhagic aspect, with high albumin level, normal chlorides and a positive Pandy and N. Appelt reaction. The prognosis was very grave, and death occurred within the first three days. Only in one case, the clinical course was more prolonged. 
The pathological study reveals and intense involvement of the venous net of the brain, which in five cases was associated with cerebral hemorrhage of various intensity.

A review of the primary or so called marantic (cachectic) type and the secondary or septic variety is presented.

\section{BIBLIOGRAFIA}

1.-FORD, F. - Jiseases of the Nervous System in In farcy, (yildtoorl and Adulescence. Charles (S Thomas. Sptriugfielel, rilitiris, 19,37 .

2--ASCHOFF, L. - Tritado de Anatomia Tatobógica.

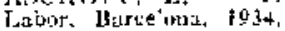

3.MLTCHEJ.J, R. G. - Venons Thrombrisis in acute hemiviegia. Arch. Dis of Childbood. 27:131. 1951.

4.-MIETTVEL, II. -.. Thrombosis sirus in Cbildren. A:t. I'entiatrica. $39: 222,1950$.

3.-VAT CLED, S.; DE BRUYXE, T. Y. Y STORSKY. M. Ci, - Thrombone du sinns Jotgitudinal superieur chez un noutrison traité par nn goutte a goutte intra.

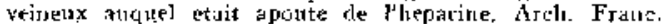
1.ert. $6: 129,1949$

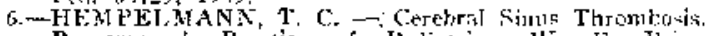
Breuemenan's Practice of I'erliatries. W. F. I'rij, Hayerstown, $194 \%$.

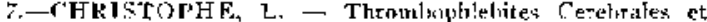
Nento-Chirurgie. l'resse Medical, $59: 119.1$. 1951.

8.-EDJTORIAJ. - Intracranial Thrombenhlehitis. The lancet, $2: 174$. 1952 ,

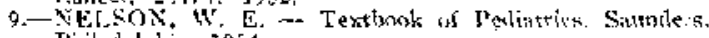
F'ilarlelitios, 1954

114. HOI.T. I. y HOW'IsAND, I, -- Trabado de l'elliatitit. Tretia, Iféjico, ]943.

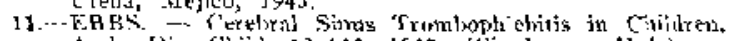

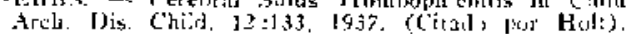

